\title{
Uberolysin: a novel cyclic bacteriocin produced by Streptococcus uberis
}

Correspondence

John R. Tagg

john.tagg@otago.ac.nz
Received 11 January 2007

Revised 22 January 2007

Accepted 23 January 2007

\author{
Ruth E. Wirawan, ${ }^{1}$ † Kara M. Swanson, ${ }^{1}$ Torsten Kleffmann, ${ }^{2}$ Ralph W. Jack ${ }^{1}$ \\ and John R. Tagg ${ }^{1}$ \\ ${ }^{1}$ Department of Microbiology and Immunology, Otago School of Medical Sciences, University of \\ Otago, PO Box 56, Dunedin, New Zealand \\ ${ }^{2}$ Centre for Protein Research (Department of Biochemistry), Otago School of Medical \\ Sciences, University of Otago, PO Box 56, Dunedin, New Zealand
}

\section{INTRODUCTION}

Streptococcus uberis, although predominantly an environmental organism (Cullen, 1969), is also commonly isolated from the lips and skin of cows, raw milk and udder tissue (Cullen, 1966; Buddle et al., 1988; Khan et al., 2003). More significantly, it is a major cause of bovine mastitis (Blowey \& Edmondson, 1995; Leigh, 1999). S. uberis belongs to the lactic acid bacteria (LAB), a group that commonly produce bacteriocins (Klaenhammer, 1993; Cotter et al., 2005).

Bacteriocins are proteinaceous antibiotics elaborated by bacteria that typically kill other bacteria of the same or

†Present address: Department of Food Science, Rutgers, The State University of New Jersey, 65 Dudley Road, New Brunswick, NJ 08901, USA.

Abbreviations: CID, collision-induced fragmentation; ESI-IT, electrospray ionization ion-trap; TFA, trifluoroacetic acid.

The GenBank/EMBL/DDBJ accession number of the sequence reported in this paper is D0650653. closely related species (Tagg et al., 1976). The majority of the characterized LAB bacteriocins are relatively small $(<10000 \mathrm{Da})$, ribosomally synthesized peptides, broadly classified as either class I, the lanthionine-containing bacteriocins (lantibiotics), or class II, the non-lanthionine-containing bacteriocins (Cotter et al., 2005; Diep \& Nes, 2002). Bacteriocins are characterized by a high level of molecular diversity and are generally presumed to have developed as mediators of inter-microbial antagonism (Diep \& Nes, 2002). Bacteriocin production is a highly complex energy-demanding process, requiring not only the synthesis of the bacteriocin precursor (prepeptide), but also the coordinated production of a set of auxiliary proteins that implement bacteriocin maturation, secretion, regulation and immunity (Nes \& Tagg, 1996; Jack et al., 1998; Sahl \& Bierbaum, 1998; Maqueda et al., 2004). Despite the considerable heterogeneity of bacteriocins, the essentially linear conformation of the backbones of their posttranslationally processed (biologically active) forms has been a common feature. More recently, however, several 
small ( $<10 \mathrm{kDa}$ ), circular bacteriocins (i.e. lacking a free Nterminus) have been described (Kalmokoff et al., 2003; Kawai et al., 1998a; Kemperman et al., 2003a; MartinezBueno et al., 1994). Although there has been a recommendation that the circular bacteriocins be considered a subgroup of the class II bacteriocins (Cotter et al., 2005), the more recent discovery of further examples of this type of molecule and recognition of the possible importance of their unique structural conformations (Kemperman et al., 2003b; Maqueda et al., 2004; Craik et al., 2003) has prompted the alternative view that they be categorized as a separate class of bacteriocin molecules (Heng \& Tagg, 2006).

Enterocin AS-48 was the first reported circular bacteriocin, and it remains the best characterized (Galvez et al., 1986, 1990; Martinez-Bueno et al., 1994, 1998; Maqueda et al., 2004). However, five more circular bacteriocins have subsequently been described: butyrivibriocin AR10 (Kalmokoff \& Teather, 1997; Kalmokoff et al., 2003), reutericin 6 (Kabuki et al., 1997; Kawai et al., 2001, 2004a), gassericin A (Kawai et al., 1998a, b, 2004b), circularin A (Kawai et al., 2004b; Kemperman et al., 2003a, b) and subtilosin A (Kawulka et al., 2004). These unusual bacteriocins have diverse characteristics; their sizes range from 3399.7 Da (subtilosin A) to $7150 \mathrm{Da}$ (enterocin AS48 ), and the number of amino acids in their leader peptides varies from three (circularin A) to 35 (enterocin AS-48). The unifying feature is the head-to-tail circularization of their peptide backbones, brought about by the direct covalent linkage of their $\mathrm{N}$ - and $\mathrm{C}$-terminal amino acids.

It is now 20 years (Tagg \& Vugler, 1986) since it was first shown that $S$. uberis strains very commonly produce bacteriocin-like inhibitory substances (BLIS) and that these exhibit a wide variety of activity spectra (Buddle et al., 1988; Jayarao et al., 1991; Klesse, 2001). A set of nine indicator bacteria is routinely used in this laboratory to detect and differentiate between the various BLIS produced by streptococci (Tagg \& Bannister, 1979). Utilizing this technique, we recently reported the identification and molecular and genetic characterization of nisin $\mathrm{U}$, a lantibiotic produced by $S$. uberis strain 42 (Wirawan et al., 2006). Analysis of the antibacterial spectrum of the purified inhibitor revealed, however, that nisin $U$ does not account for the entire inhibitory activity displayed by strain 42 against the standard indicator bacteria. Here we report the isolation, purification and characterization of uberolysin, a novel member of the circular bacteriocins that is also produced by S. uberis strain 42 .

\section{METHODS}

Bacterial strains, media and culture conditions. All bacterial strains used in this study were obtained from our laboratory culture collection. The set of nine standard bacteriocin indicator strains (I1I9) have been described previously (Tagg \& Bannister, 1979). Routine subculturing was carried out on BACa (Columbia Agar Base, CAB; Becton Dickinson) supplemented with $5 \%$ (v/v) human blood and $0.1 \%(\mathrm{w} / \mathrm{v}) \mathrm{CaCO}_{3}$ or in Todd-Hewitt broth (THB;
Becton Dickinson). All of the solid media contained $1.5 \%(\mathrm{w} / \mathrm{v})$ bacteriological agar (Scientific Supplies Ltd). In general, incubation was for $18 \mathrm{~h}$ at $37^{\circ} \mathrm{C}$ in $5 \% \mathrm{CO}_{2}$-enriched air.

Activity assays. The spectrum of inhibitory activity of the uberolysin producer strain (S. uberis 42) and its uberolysin-negative derivative (S. uberis ublyko1) were compared by deferred antagonism against the nine standard indicators (Tagg \& Bannister, 1979) and other representative strains. In addition, the activities of purified uberolysin and nisin $U$ were tested against the same strains by spot assay, as previously described (Wescombe \& Tagg, 2003). The inhibitory activity of uberolysin was examined by both solid and liquid culture-based methods. For solid media experiments, the indicator strain A5 was propagated in $10 \mathrm{ml} \mathrm{THB}\left(37^{\circ} \mathrm{C}, 18 \mathrm{~h}\right)$, following which the cells were harvested by centrifugation, and washed twice in saline solution. The cell pellet was resuspended in $0.5 \mathrm{ml}$ deionized water, added to $100 \mathrm{ml}$ molten Todd-Hewitt agar (THA) and $15 \mathrm{ml}$ plates were poured. Drops $(20 \mu \mathrm{l})$ of uberolysin preparations were spotted onto the agar, and allowed to dry. Following incubation at $37^{\circ} \mathrm{C}$, the agar was examined for zones of clearance of bacterial growth. This same method was used to determine the activity of uberolysin on A5 cell suspensions in which the cells had been killed either by pre-mixing the cell suspension $1: 1(\mathrm{v} / \mathrm{v})$ with chloroform, or by heating at $65^{\circ} \mathrm{C}$ for $20 \mathrm{~min}$. Lysozyme $\left(1 \mathrm{mg} \mathrm{ml}{ }^{-1}\right)$ was spotted onto the agar as a positive control of cell lysis. For liquid culture assays, eight sterile cuvettes, each containing $2 \mathrm{ml} \mathrm{THB}$, were inoculated with $200 \mu \mathrm{l}$ of an $18 \mathrm{~h}$ culture of Lactococcus lactis A5. One cuvette (control) remained untreated, and along with the seven remaining cuvettes was incubated at $37^{\circ} \mathrm{C}$. The additional seven cuvettes were each supplemented with $20 \mu \mathrm{l}$ purified uberolysin (final titre $1 \mathrm{AU} \mathrm{m}^{-1}$ ) at 10, 15, 30, 60, 90, 120 or $210 \mathrm{~min}$, respectively. The $\mathrm{OD}_{585}$ was monitored and readings taken after 10, 15, 30, 60, 90, 120, 150, 165, 180, 210, 240, 420 and $480 \mathrm{~min}$. The effect of uberolysin on the viable cell count (c.f.u. $\mathrm{ml}^{-1}$ ) of $L$. lactis $\mathrm{A} 5$ was determined by plating and enumerating appropriate dilutions at 10, 30, 60, 90, 120 and 150 min after addition of purified uberolysin to a mid-exponential-phase culture.

Extraction and purification of uberolysin. Lawn cultures of $S$. uberis 42 were grown on $\mathrm{CAB}$ plates and, following incubation $(18 \mathrm{~h}$, $37^{\circ} \mathrm{C}$ ), were placed at $-80^{\circ} \mathrm{C}$ for $4-6 \mathrm{~h}$, and subsequently thawed at room temperature. The exudate was collected, the cells and agar debris removed by centrifugation $\left(15300 \mathrm{~g}, 15 \mathrm{~min}, 4^{\circ} \mathrm{C}\right)$, and the supernatant analysed for inhibitory activity by spot assay. Solid ammonium sulfate was added to the extract from the $\mathrm{CAB}$ cultures to achieve $50 \%$ saturation at $4{ }^{\circ} \mathrm{C}$, and the mixture was stirred gently for $18 \mathrm{~h}$. The inhibitor-containing precipitate was then harvested by centrifugation $\left(15300 \mathrm{~g}, 30 \mathrm{~min}, 4^{\circ} \mathrm{C}\right)$ and redissolved in $100 \mathrm{ml}$ deionized water. After clarification by centrifugation, the supernatant fluid was passed through a Millex-HA $0.45 \mu \mathrm{m}$ pore filter unit (Millipore) to sterilize the preparation. This crude material was then diluted 10fold with $25 \mathrm{mM}$ 2-morpholinoethanesulfonic acid (MES; SigmaAldrich), pH 5.8 and was subsequently loaded onto a CM-Macroprep column ( $25 \mathrm{ml}$, Bio-Rad), equilibrated with the same buffer, that was connected to an Äkta Explorer (GE Healthcare Biosciences). The unbound material was washed out in 20 column volumes of the same buffer and the column was then developed with a linear gradient of $0-0.5 \mathrm{M} \mathrm{NaCl}$ (in $25 \mathrm{mM}$ MES, pH 5.8) at a constant flow rate of $3.0 \mathrm{ml} \mathrm{min}{ }^{-1}$ over 30 column volumes. Fractions $(12.5 \mathrm{ml})$ were examined for inhibitory activity against $L$. lactis A5 by spot assay (see below). Active fractions were pooled, concentrated by ammonium sulfate precipitation $\left(80 \%\right.$ saturation at $\left.4{ }^{\circ} \mathrm{C}\right)$, and $0.5 \mathrm{ml}$ samples were fractionated by gel permeation chromatography on a Superdex 75 10/ 300 GL (GE Biosciences Healthcare) column in $150 \mathrm{mM}$ ammonium hydrogen carbonate (BDH Laboratory Supplies), $\mathrm{pH}$ 8.0, at a constant flow rate of $0.5 \mathrm{ml} \mathrm{min}{ }^{-1}$. Fractions exhibiting inhibitory activity were further pooled and finally purified by reverse-phase (RP) HPLC 
using a $\mathrm{C}_{8}$ column (Brownlee RP-300 Aquapore, $7 \mu \mathrm{m}, 30 \mathrm{~nm}$ pore, $4.6 \times 40 \mathrm{~mm}$ [Alltech]) in a linear gradient of $10-90 \%(\mathrm{v} / \mathrm{v})$ aqueous 2-propanol containing $0.1 \%(\mathrm{v} / \mathrm{v})$ trifluoroacetic acid (TFA) over

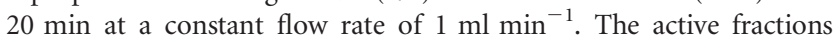
(purified uberolysin) were pooled and stored frozen $\left(-20^{\circ} \mathrm{C}\right)$ and were further used for mass spectrometry (MS) analysis and N-terminal amino acid sequencing.

Hydrolysis of uberolysin with chymotrypsin. Digestion with chymotrypsin (Sigma) was performed at an enzyme:substrate ratio of approximately $1: 40$ in $150 \mathrm{mM}$ ammonium bicarbonate (BDH) $\mathrm{pH} 8.5,37^{\circ} \mathrm{C}$ for $18 \mathrm{~h}$. Separation of the digested products was by RP-HPLC using a $\mathrm{C}_{18}$ column (Gemini, $5 \mu \mathrm{m}, 11 \mathrm{~nm}$ pore, $2 \times 50 \mathrm{~mm}$ [Phenomenex]), with a gradient of $0-100 \%$ buffer B $(90 \%$, $\mathrm{v} / \mathrm{v}$, aqueous acetonitrile containing $0.1 \%, \mathrm{v} / \mathrm{v}$, TFA) over $20 \mathrm{~min}$. Analysis of the individual peptide-containing fractions was followed, where appropriate, by mass spectrometry and $\mathrm{N}$-terminal sequencing.

Protein electrophoresis and agar overlay assays. Peptide samples were mixed with an equal volume of Tricine sample buffer (Bio-Rad), then heated at $100^{\circ} \mathrm{C}$ for $7 \mathrm{~min}$ (and immediately placed on ice) prior to loading onto a Novex $16 \%$ Tricine (Invitrogen Life Technologies) gel with a MultiMark multi-coloured protein standard (Invitrogen). The gels were run in a XCell SureLock Mini-Cell apparatus (Invitrogen) using running buffer made from a $10 \times$ Tris/ Tricine/SDS stock (Bio-Rad) according to the manufacturer's instructions, following which they were stained with $0.1 \%(\mathrm{w} / \mathrm{v})$ Coomassie brilliant blue G-250 (Bio-Rad) in $40 \%(\mathrm{v} / \mathrm{v})$ methanol and $10 \%(\mathrm{v} / \mathrm{v})$ acetic acid, destained $(25 \%, \mathrm{v} / \mathrm{v}$, methanol, $10 \%$, $\mathrm{v} / \mathrm{v}$, acetic acid) and photographed. For activity assays, a portion of the gel containing a marker and peptide sample was excised prior to staining, soaked in several changes of deionized water for $18 \mathrm{~h}$, and subsequently overlaid with $15 \mathrm{ml}$ molten THA containing washed $L$. lactis A5 cells (from a $3 \mathrm{ml}$ THB culture).

MS and $\mathbf{N}$-terminal amino acid sequence analyses. MS was conducted by matrix-assisted laser desorption ionization-time of flight (MALDI-TOF) or by electrospray ionization ion-trap (ESI-IT). MALDI-TOF MS was carried out using a Finnigan LaserMAT 2000 (Thermo BioAnalysis) mass analyser, in which $0.5-1 \mu \mathrm{l}$ samples of peptide dissolved in $60 \%(\mathrm{v} / \mathrm{v})$ aqueous acetonitrile containing $0.1 \%(\mathrm{v} / \mathrm{v})$ TFA were applied to stainless steel slides together with $0.5 \mu \mathrm{l}$ of $10 \mathrm{mg} \mathrm{ml}^{-1} \alpha$-cyano-4-hydroxycinnamic acid and air-dried prior to analysis. Positive-mode ESI-IT mass spectra were accumulated by direct introduction of peptide samples (dissolved in $30 \%$, $\mathrm{v} / \mathrm{v}$, aqueous acetonitrile containing $0.2 \%, \mathrm{v} / \mathrm{v}$, formic acid) via a syringe pump into the ESI interface of a LCQ Deca mass-spectrometer (ThermoFinnigan). Mass spectra were processed and analysed using the manufacturer's software. N-terminal amino acid sequencing of peptides was performed with a model 492 ABI Procise HT pulsed gas-liquid microsequencer (Applied Biosystems). Samples for automated Edman degradation were applied to a TFA-treated glass fibre support. MS and N-terminal amino acid sequencing were carried out at the Protein Microchemistry Facility (Department of Biochemistry, University of Otago).

\section{In-gel tryptic digestion of uberolysin and MALDI tandem} TOF MS analyses of digestion products. Using Tris-TricineSDS-PAGE (Invitrogen), approximately $10 \mu \mathrm{g}$ uberolysin was separated in three separate lanes and the uberolysin bands were excised from the gel, pooled and subjected to in-gel tryptic digestion essentially according to the method of Shevchenko et al. (1996). The uberolysin bands were digested with $2 \mu$ g proteomic-grade trypsin (Sigma-Aldrich) and, after digestion and lyophilization of the gel eluates, the peptides were resuspended in $10 \mu \mathrm{l} \%$ (v/v) aqueous acetonitrile containing $0.1 \%(\mathrm{v} / \mathrm{v})$ TFA and purified by ZipTip (Millipore) extraction according to the manufacturer's instructions. Prior to transfer to the MALDI plate, standard peptides were 'spiked' into the ZipTip eluate to facilitate internal MS calibration. The sample volume was reduced to $2-3 \mu \mathrm{l}$ by vacuum evaporation and then spotted onto the MALDI plate by pipetting three $0.8 \mu \mathrm{l}$ aliquots to a single target spot. An aliquot $(0.8 \mu \mathrm{l})$ of matrix $\left(10 \mathrm{mg} \mathrm{ml}^{-1} \alpha-\right.$ cyano-4-hydroxycinnamic acid dissolved in $50 \%$ [v/v] aqueous acetonitrile containing $0.1 \%[\mathrm{v} / \mathrm{v}]$ TFA) was immediately mixed directly on the plate and the sample/matrix mixture was air-dried. The tryptic digest of uberolysin was analysed on a model 4800 MALDI tandem TOF Analyser (Applied Biosystems). All spectra were acquired in positive-ion mode with 800 laser pulses for MS acquisition and 2000 laser pulses for MS/MS collision-induced fragmentation (CID) analyses. MS/MS CID spectra were acquired in the $1 \mathrm{kV}$ mode using air as the collision gas. The instrument's default calibration parameters for MS and MS/MS mode were updated by acquiring data on six calibration spots. After the acquisition of a full MS spectrum on the sample and internal calibration based on the standard peptide peaks, the precursor ion with $\mathrm{m} / \mathrm{z} 1878.01$ was selected for CID analysis. Using the Mascot search engine (http://www.matrixscience.com) the MS/MS data obtained were searched against a modified SWISS-PROT sequence database (downloaded in June 2006), containing two separate entries based on a cyclic uberolysin sequence that had been arbitrarily linearized, either between Trp70 and Leu1 (i.e. ${ }^{\mathrm{H}-} \mathrm{Leu} 1 \rightarrow \operatorname{Trp} 70^{-\mathrm{OH}}$ ) or between Ile28 and Ile29 (i.e. ${ }^{\mathrm{H}-} \mathrm{Ile} 29 \rightarrow \mathrm{Ile} 28^{-\mathrm{OH}}$ ).

Mutagenesis of S. uberis strain 42 using Tn916. Tn916 mutagenesis was carried out essentially as described previously (Nida \& Cleary, 1983). Samples $(250 \mu \mathrm{l})$ of $3 \mathrm{ml}$ cultures of a streptomycinresistant $\left(\mathrm{Str}^{\mathrm{r}}\right)$ derivative of $S$. uberis strain 42 (recipient) and Enterococcus faecalis CG110 (donor) were inoculated into $5 \mathrm{ml}$ THB supplemented with $2 \%(\mathrm{w} / \mathrm{v})$ neopeptone (Difco) and $1 \%(\mathrm{w} / \mathrm{v})$ yeast extract (Becton Dickinson) (THNY). The cells were immediately deposited onto a $0.45 \mu \mathrm{m}$ MF-membrane filter (Millipore), which was then transferred onto THNY blood agar. Following incubation the cell growth was harvested, and plated on THN agar containing $1 \mathrm{mg}$ Str $\mathrm{ml}^{-1}$ and $10 \mu \mathrm{g} \mathrm{Tet} \mathrm{ml}^{-1}$. Potential transconjugant colonies were tested for inhibitory activity by toothpick transfer onto BACa and incubation. The colony growth was then removed; the plates were subsequently sterilized with chloroform vapour, and seeded with lawns of Micrococcus luteus T-18 or L. lactis A5. Putative transconjugants containing Tn916 disruption of the uberolysin locus were recognized by their inability to inhibit the growth of L. lactis A5, while retaining activity (due to nisin U: Wirawan et al., 2006) against $M$. luteus T-18. One mutant, designated ublykol (confirmed to be $S$. uberis by $16 \mathrm{~S}$ rDNA sequencing), was selected for further studies.

Identification and characterization of the uberolysin biosynthetic locus. Conventional molecular techniques and DNA detection assays used were essentially as described by Sambrook \& Russell (2001). DNA was extracted using the $\mathrm{LiCl}$ method described previously (Wirawan et al., 2006). All primers were synthesized by Invitrogen and are listed in Table 1. Identification of the Tn916 insertion site in three potential mutants, and subsequent amplification of the uberolysin locus, was achieved by a series of inverse PCR reactions (Sambrook \& Russell, 2001). Chromosomal DNA digested with HindIII and NdeI, and ligated with T4 ligase (all supplied by Roche), was used as template DNA. Selected PCR amplicons were purified from agarose gel slices using the QiaQuick gel extraction kit (Qiagen), and submitted for nucleotide sequencing. All inverse PCR experiments utilized the Expand Long Template PCR Kit (Roche), using conditions recommended by the manufacturer. Nucleotide sequencing reactions were carried out at the Allan Wilson Centre Genome Service (Massey University, New Zealand), and data were assembled and analysed using the Lasergene sequence analysis software package (DNASTAR). Homology searches used the appropriate BLAST algorithms (Altschul et al., 1997) available at the National Center for Biotechnology Information (NCBI) website (http://www.ncbi.nlm.nih.gov), and against the genome sequence of $S$. uberis available on the Sanger 
Table 1. Oligonucleotides used in this study

\begin{tabular}{|llc|}
\hline Primer name & \multicolumn{1}{c|}{ Primer sequence } & Location \\
\hline 1536 & GTTGCGGCCGCGATAAAGTGTGATAAGTCCAG & $64-36^{*}$ \\
1545 & ATAGCGGCCGCTTAGCTCATGTTGATGCGG & $12136-12164^{*}$ \\
1537 & TACGCGGCCGCACATAGAATAAGGCTTTACG & $17954-17984^{*}$ \\
1546 & CCTGCGGCCGCGCTTCCTAATTCTGTAATC & $12238-12209^{*}$ \\
TetMFwd & TTTTTAGGAGGGCTTAGTTT & $11940-11959^{*}$ \\
TetMRev & CATATGTCCTGGCGTGTCT & $12359-12341^{*}$ \\
ublylocUSinvR & TGTTTCTATCTTTGGTGGCATTGTTGTT & $679-706 \dagger$ \\
ublRUSinvL & TGCCATGTTTATTTGCTGCATCAATGGA & $1289-1262 \dagger$ \\
ublRFwd & TGGTCTTTTTTAACTGTGTTTGTCA & $1361-1385 \dagger$ \\
ublyUSinvR & ACATGTTACTACCTCACAAATAAAT & $2135-2159 \dagger$ \\
ublyHind3invL & AGGTAGTAACATGTTATCGGTATT & $2148-2125 \dagger$ \\
ublyFwd2 & GGGATTTAACATGGACATTTTATTAG & $2428-2453 \dagger$ \\
ublyRev2 & AGCTCCTTTATCAAATTATTAGTCG & $2749-2725 \dagger$ \\
6.1 invL & TCGTGGAATTTCTAACTTGATA & $2965-2944 \dagger$ \\
6.1 invR & TCGAAACACCCTTGGATAGAATG & $3049-3071 \dagger$ \\
ublBDSinvR & GGACATATATTATGCCTAACTTCACA & $4097-4122 \dagger$ \\
ublyNdeinvL & AGCAATAATTTAATAGCCACACCA & $4620-4597 \dagger$ \\
11.1 invL & GTCGATATTCTCAAAACCACTTA & $5181-5159 \dagger$ \\
11.1 invR & TTTGGAAGAAAGTCGTGATGAG & $5202-5223 \dagger$ \\
11 seq2R & GTCGTGATGAGGTACTAGATAACA & $5213-5236 \dagger$ \\
ublyUSinvL & TCCAAATTACTCATCAGTTCATCT & $5378-5355 \dagger$ \\
ublylocFwd & AGAAATGATGGATGGTCGTGTCAAG \\
ublylocRev & ATGGTTGGTTGATGGGTGATACGA & \\
\hline & & \\
\hline
\end{tabular}

${ }^{\star}$ Primers correspond to Tn916 sequence (GenBank accession no. U09422). Primers numbered in reverse are located on the complementary strand.

$\dagger$ Locations of primers are based on GenBank accession number DQ650653. Primers numbered in reverse are located on the complementary strand.

Institute website (http://www.sanger.ac.uk/Projects/S_uberis/). Two additional primers, ublylocFwd and ublylocRev, were designed based on homologies to the S. uberis genome sequence, and used in PCR to generate a $7 \mathrm{~kb}$ contiguous sequence encompassing the entire uberolysin biosynthetic locus (accession no. DQ650653).

Distribution of the uberolysin structural gene (ub/A) in S. uberis. The primers ublyFwd2 and ublyRev2 (Table 1) were used to amplify the uberolysin structural gene, ublA. DNA extracted from 45 strains of S. uberis was applied to a nylon membrane (Hybond-N+; GE Healthcare Biosciences) using a Dot Blot apparatus (Bio-Rad), and probed with digoxigenin-dUTP (Roche)-labelled $u b l A$ as described previously (Wescombe \& Tagg, 2003). The presence of $u b l A$ in other strains was also established by colony PCR. A single colony of each strain to be tested was added to a mix containing each primer, nucleotides, buffer and HotMaster Taq (Eppendorf). PCR consisted of an initial heating step of $94^{\circ} \mathrm{C}$ for $5 \mathrm{~min}$, followed by 30 cycles of $94^{\circ} \mathrm{C}$ for $30 \mathrm{~s}$, annealing at $50{ }^{\circ} \mathrm{C}$ for $30 \mathrm{~s}$, and elongation at $65^{\circ} \mathrm{C}$ for $30 \mathrm{~s}$.

\section{RESULTS AND DISCUSSION}

\section{A second novel antibacterial agent is produced by $S$. uberis 42}

Our previous studies have shown that $S$. uberis 42 produces a lantibiotic, named nisin $U$, which in its purified form inhibits the growth of each of the bacterial strains in our set of nine standard bacteriocin indicators (I1 to I9) with the exception of I4 (Wirawan et al., 2006). Interestingly, this indicator is a $S$. uberis strain that also produces (and is therefore specifically immune to) nisin U. Since strain 42 strongly inhibits all nine standard indicators in the deferred antagonism test, we hypothesized that this strain produces an additional bacteriocin (or bacteriocins) inhibitory to (at least) indicator I4. Subsequently, we have found (data not shown) that the nisin Z-producing strain, L. lactis A5, is superior as an indicator since, in addition to displaying cross-immunity to nisin U (Wirawan et al., 2006), it is more sensitive than S. uberis I4 to the non-nisin U antimicrobial agent(s).

Freeze-thaw extracts of $\mathrm{CAB}$ agar lawn cultures of strain 42 yielded considerable inhibitory activity (titre $1.9 \times 10^{4} \mathrm{AU} \mathrm{ml}^{-1}$ ) against L. lactis $\mathrm{A} 5$, and these extracts were used as the starting material for purification of the bacteriocin(s). Cation-exchange chromatography localized the anti- $L$. lactis A5 inhibitory activity to ten $(12.5 \mathrm{ml})$ fractions eluting in the range of $0.1-0.15 \mathrm{M} \mathrm{NaCl}$, whilst gel permeation followed by RP-HPLC yielded a single peak containing inhibitory activity. From a sample of this purified material (named uberolysin), a single component 
with a mass of $7047.97 \pm 0.09 \mathrm{Da}$ (mean $\pm \mathrm{SD}, n=3$ ) was detected by ESI-IT MS. Initial attempts to obtain the amino acid sequence by Edman degradation were unsuccessful, indicating that access to the $\mathrm{N}$-terminal residue was blocked.

In contrast to the mass obtained by ESI-IT MS, Tris-TricineSDS-PAGE combined with either Coomassie blue staining or agar overlay indicated a band corresponding to approximately $4500 \mathrm{Da}$ (Fig. 1). Interestingly, anomalous behaviour of some other bacteriocins during electrophoretic separation has been reported previously. For example, trypsin and lysylendopeptidase digestion (linearization) altered the migration behaviour of the circular bacteriocin gassericin A on SDS-PAGE from an apparent molecular mass of $3800 \mathrm{Da}$ (cyclic) to the expected size of $5600 \mathrm{Da}$ (Kawai et al., 2003). Similarly, the putative cyclic peptide acidocin B (98\% similar to gassericin A) separated as a band of 2400 Da by SDS-PAGE, in spite of having a predicted size of $5800 \mathrm{Da}$ (Kawai et al., 1998a). Recently, establishment of the three-dimensional structure of the prototype circular bacteriocin enterocin AS-48 by NMR showed it to be a compact molecule, consisting of five $\alpha$-helices, with the hydrophobic side chains forming the core of the protein (Maqueda et al., 2004). Thus, the highly constrained (a)

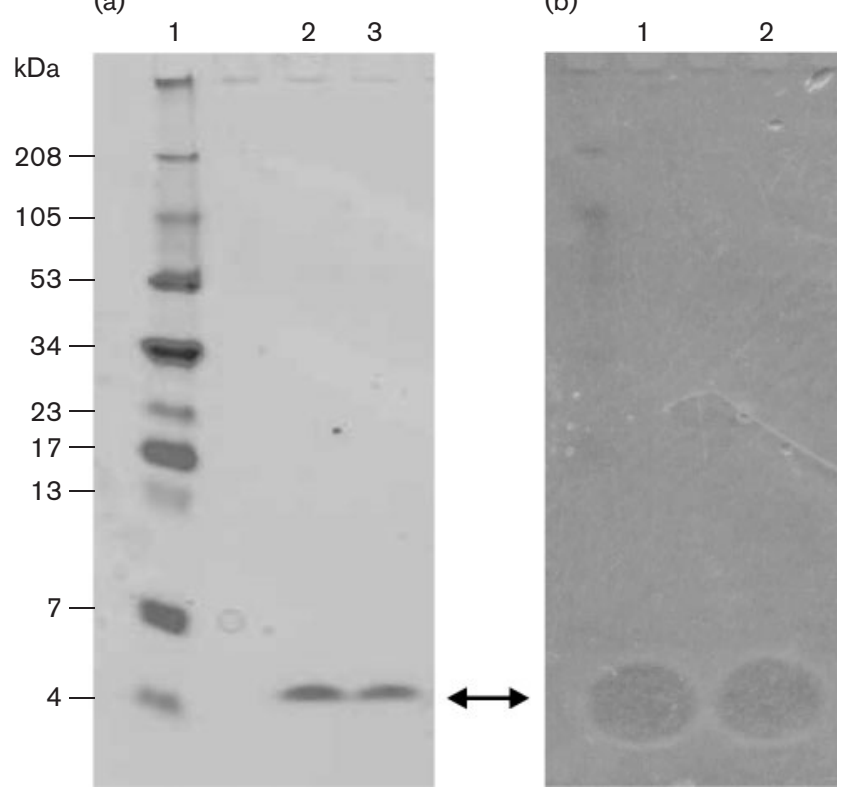

Fig. 1. SDS-PAGE analysis of uberolysin. (a) Duplicate samples of uberolysin (lanes 2 and 3 ) were separated by $16 \%$ Tris-Tricine SDS-PAGE and stained with Coomassie blue; lane 1 contains markers of the indicated molecular mass. (b) Antimicrobial activity of electrophoresed uberolysin was assessed by overlaying a washed portion of gel in which duplicate samples of uberolysin had been separated (lanes 1 and 2) with THA containing the sensitive indicator bacterium $L$. lactis A5. In both panels, the presence of uberolysin is indicated by the double-headed arrow. structure of the circular bacteriocins has been suggested to account for their unusual migration behaviour on SDSPAGE gels (Kalmokoff et al., 2003; Kawai et al., 2004b).

\section{Identification of the uberolysin structural gene ublA}

In order to identify the genetic determinant(s) encoding uberolysin biosynthesis, we screened for transconjugants lacking antimicrobial activity against $L$. lactis A5 following Tn916 mutagenesis. Of 96 potential transconjugants screened, 10 were presumptively identified as uberolysindeficient mutants because they failed to inhibit the indicator L. lactis A5 and each was P-type 737 (i.e. they were active against all but indicator I4 of the nine standard indicators and therefore displayed inhibition patterns typical of nisin $U$ production in the absence of uberolysin: Wirawan et al., 2006). Inverse PCR products obtained using DNA from three mutants (ublyko1, ublyko7 and ublyko10) showed similar profiles by gel electrophoresis, indicating that there may be a preferential site of insertion of $\mathrm{Tn} 916$ within the uberolysin locus (data not shown). Subsequent sequencing and BLAST analysis of DNA flanking the transposon insertion site returned a single homology to the product of $p B t 137$, a putative maturation factor for a peptide antibiotic encoded on pBtoxis of Bacillus thuringiensis subsp. israelensis, which itself has weak similarity with the prototype cyclic bacteriocin enterocin AS-48 (Berry et al., 2002). The strain 42 gene targeted by Tn916 was therefore designated $u b l B$ (Fig. 2).

Southern blot analysis indicated that only a single copy of Tn 916 was present in the DNA of S. uberis strain ublykol. Further sequence analysis of this strain revealed a $231 \mathrm{bp}$ ORF located upstream of $u b l B$ that we designated $u b l A$, the putative uberolysin structural gene (Fig. 2a). The primary translation product of $u b l A$ is predicted to be a 76 amino acid polypeptide (Fig. 2b). In order to confirm that $u b l A$ was indeed the structural gene encoding the precursor of the peptide isolated, we used enzyme-mediated hydrolysis to obtain the amino acid sequence of uberolysin. Although chymotrypsin digestion of uberolysin for $6 \mathrm{~h}$ failed to release any peptide fragments, several peaks were detected by RP-HPLC in digests incubated for $18 \mathrm{~h}$. The most prominent of these corresponded to an $818 \mathrm{Da}$ peptide (estimated by MALDI-TOF MS) eluting at approximately $30 \%$ acetonitrile, and amino acid sequence analysis of this fragment yielded the sequence ${ }^{\mathrm{H}-} \mathrm{KAQAVIW}^{-\mathrm{OH}}$ (calculated mass $815 \mathrm{Da}$ ). This sequence corresponds precisely with the predicted C-terminus $\left(\mathrm{Lys}^{70}-\operatorname{Trp}^{76}\right)$ of UblA, confirming that $u b l A$ encodes the uberolysin precursor. Interestingly, the mean mass calculated following removal of the six amino acids at the $\mathrm{N}$-terminus of the uberolysin prepeptide (7066.30) is approximately $18 \mathrm{Da}$ (the mass of water) more than that measured by ESI-IT MS (mean mass = $7047.97 \pm 0.09$ ), indicating that uberolysin is post-translationally modified. 


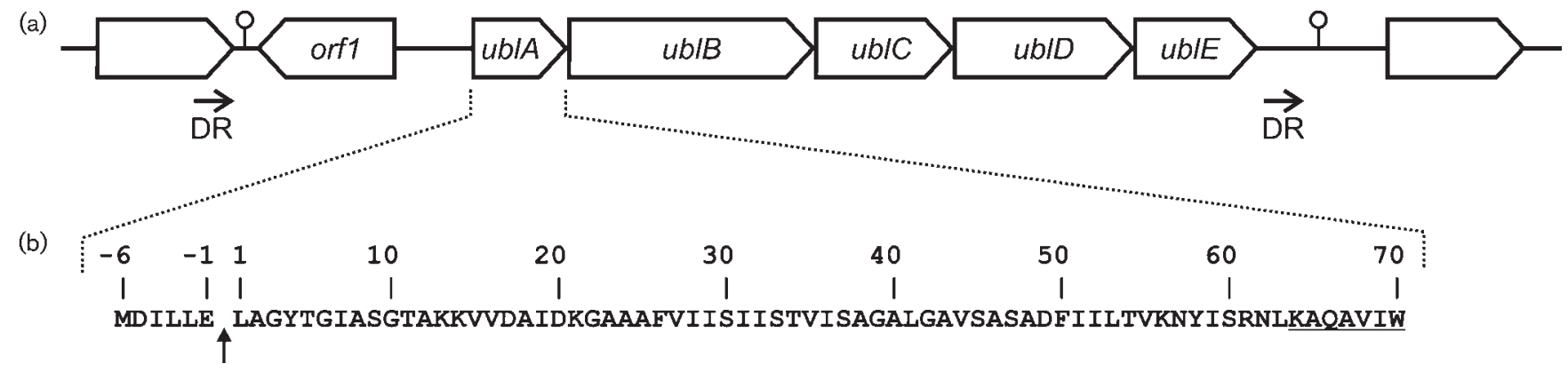

Fig. 2. Characterization of the genetic elements encoding uberolysin production in S. uberis strain 42. (a) The organization of the $u b /$ biosynthetic locus and immediate flanking chromosomal regions in $S$. uberis strain 42 . Open block arrows indicate ORFs and their relative orientation. Horizontal arrows indicate the location of the direct repeats (DR). (b) The deduced $u b / A$ primary translation product with the putative cleavage site of the leader peptide indicated by the vertical arrow; the sequence obtained from the fragment of uberolysin generated by chymotrypsin digestion is underlined.

\section{Uberolysin is a cyclic bacteriocin}

Since uberolysin was refractory to Edman degradation, migrated anomalously by SDS-PAGE, and appeared to be approximately $18 \mathrm{Da}$ smaller than would be predicted after removal of the first 6 amino acids of UblA, we hypothesized that this bacteriocin may be cyclic (i.e. dehydrated through formation of an additional peptide bond). In order to confirm this feature we firstly assessed the monoisotopic mass of uberolysin by MALDI-TOF MS. As was observed for the mean mass measured by ESI-IT MS, the measured monoisotopic mass of uberolysin (7043.94) was 18 Da less than that calculated for a linear peptide (7061.96), but within 0.01 mass units of that calculated for a cyclic derivative of UblA after removal of the first 6 amino acids (7043.95). Secondly, we carried out in-gel tryptic digestion of uberolysin and obtained a fragment of $[M+\mathrm{H}]^{+}=1878.01$ that was unique in that it would not be predicted to arise from a linear sequence spanning Leu ${ }^{1}-$ $\operatorname{Trp}^{70}$; it could however result from digestion of cyclic uberolysin and represent a contiguous peptide sequence spanning $\mathrm{Ala}^{65}$ to $\mathrm{Lys}^{13}$. This result not only implies that uberolysin is cyclic but indicates it is a head-to-tail monocycle. Finally, in order to confirm this and to rule out the possibility of side-chain modifications as the source of mass discrepancy, the $[\mathrm{M}+\mathrm{H}]^{+}=1878.01$ tryptic peptide was analysed by CID-MS/MS. A search of the modified SWISS-PROT database using the Mascot search engine (see Methods) unambiguously matched only a single sequence of ${ }^{\mathrm{H}-}$ AQAVIWLAGYTGIASGTAK ${ }^{-\mathrm{OH}}$. This sequence corresponds exactly to that calculated for the sequence of a 19 amino acid polypeptide fragment generated from trypsinmediated hydrolysis of the peptidyl bonds between Lys ${ }^{64}$ and $\mathrm{Ala}^{65}$, and between $\mathrm{Lys}^{13}$ and $\mathrm{Lys}^{14}$, and includes a peptide bond between $\operatorname{Trp}^{70}$ and Leu ${ }^{1}$ of uberolysin. Taken together, these results indicate that uberolysin is a head-totail monocycle and contains no further post-translational modifications.

\section{Sequence analysis of the region encoding the putative uberolysin locus}

Tn916 mutagenesis enabled detection of the uberolysin locus, and sequence assembly of a series of products generated by inverse PCR identified a $7 \mathrm{~kb}$ contiguous region encompassing the putative uberolysin locus in strain 42. Sequence analyses and BLAST searches of the sequence surrounding the structural gene $u b l A$ revealed six ORFs potentially involved in the production of uberolysin (Fig. 2). Detailed characterization of the loci involved in circular bacteriocin biosynthesis have been described for enterocin AS-48 (Diaz et al., 2003; Martinez-Bueno et al., 1998), circularin A (Kemperman et al., 2003a), butyrivibriocin AR10 (Kalmokoff et al., 2003) and subtilosin A (Zheng et al., 2000). The minimal region encoding circularin A biosynthesis, processing and secretion in a heterologous host was encompassed by five genes, cirABCDE, although the entire locus contains 11 genes (Kemperman et al., 2003a). Similarly, enterocin AS-48 production and immunity was initially thought to be encoded by as-48ABCC $D_{1} D_{1}$ (Martinez-Bueno et al., 1998), and the butyrivibriocin locus reportedly consists only of bviABCDE (Kalmokoff et al., 2003). With the exception of the structural genes and those encoding $A B C$ transporters (as-48D, $\operatorname{cir} B, b v i B$ ), the functions of the remaining genes remain unclear.

The genes within the uberolysin locus were designated orf1, $u b l A, u b l B, u b l C, u b l D$ and $u b l E$, and the sizes of the gene products and their putative functions based on BLAST homologies are presented in Table 2. orf1, located $303 \mathrm{bp}$ upstream of ublA but in the opposite transcriptional orientation, encodes a protein with conserved-domain homology to the LytR family of response regulators. However, orf1 appears to be an orphan response regulator, since there is no corresponding ORF with homology to sensor kinases in its vicinity. A number of the LytR family of 
Table 2. Characteristics of predicted proteins specified by the $S$. uberis $42 \mathrm{ub}$ locus

\begin{tabular}{|c|c|c|c|c|c|}
\hline \multirow[t]{2}{*}{ ORF } & \multirow[t]{2}{*}{ ORF size (bp) } & \multicolumn{4}{|c|}{ Deduced protein } \\
\hline & & Size $(k D a)$ & pI & Homology (\% identity, \% similarity) & Proposed function \\
\hline ubla & 231 & 7.8 & 8.5 & Putative peptide antibiotic $(43,60)$ & Uberolysin prepeptide \\
\hline$u b l B$ & 1608 & 63.5 & 9.5 & CirB $(23,43)$ & Circularization of bacteriocin \\
\hline$u b l C$ & 546 & 21.3 & 8.7 & $\begin{array}{l}\text { Integral membrane protein, possible accessory } \\
\text { factor in peptide antibiotic secretion }(35,58)\end{array}$ & Accessory factor for transport \\
\hline$u b l D$ & 657 & 25.5 & 4.8 & $\operatorname{CirD}(35,59)$ & Transport of bacteriocin \\
\hline ublE & 267 & 10.2 & 9.2 & No homology & Immunity peptide \\
\hline
\end{tabular}

regulators have been implicated in bacteriocin regulation, for example BlpR from Streptococcus pneumoniae (Knutsen et al., 2004) and EntR from Enterococcus faecium (Hickey et al., 2003). Genes encoding putative response regulators belonging to the LytR family are also found upstream of both the butyrivibriocin (Kalmokoff et al., 2003) and circularin (Kemperman et al., 2003a) structural genes. Although no orphan response regulators have yet been implicated in bacteriocin production, their occurrence is not unusual, and they have been predicted to have a number of (sometimes essential) functions (Ulijasz et al., 2004; Hutchings et al., 2004; Hancock \& Perego, 2002; de Greeff et al., 2002; Haydel \& Clark-Curtiss, 2004). This putative regulator may be essential for uberolysin production, as S. uberis strain 0140J, which encodes only a truncated copy of the putative regulator (see below), yields no detectable uberolysin. On the other hand, strain 233, which does produce uberolysin, has an intact copy of ORF1.

The uberolysin precursor (UblA) is a 76 amino acid polypeptide encoded by $u b l A$ from which uberolysin is derived. UblA appears to have a very short 'leader' peptide, consisting of only six amino acids, and circularization occurs between Leu7 and Trp76 of UblA. This short leader peptide is similar to that reported for circularin A, where only three $\mathrm{N}$-terminal amino acids of the prepeptide are removed during the formation of the active molecule (Kemperman et al., 2003b). This is in contrast to other circular bacteriocins, where the primary translation products have leader sequences of 22, 33 and 35 residues for butyrivibriocin AR10, gassericin A and enterocin AS-48, respectively. The function of the leader peptides, the method of leader cleavage and the steps involved in circularization of the peptide remain unknown. A model has been proposed for enterocin AS-48, whereby the dedicated ABC transporter, AS-48D (combined with $\mathrm{AS}-48 \mathrm{C}_{1}$ ), and an integral membrane protein, AS-48B, induce formation of a pore specifically to facilitate head-to-tail cyclization of the preAS-48 peptide, concomitant with export of the molecule (Maqueda et al., 2004). CirBD are the equivalents of AS48BD in the circularin operon, and it is also proposed that CirC may be important in maturation of circularin (Kemperman et al., 2003a). Based on their amino acid sequence similarities, $u b l B D$ are likely to encode the transporter complex, whereas UblC appears to be the equivalent of the AS-48C and CirC proteins.

As with the lantibiotic class of bacteriocins (Jack et al., 1998; Sahl \& Bierbaum, 1998), two principal mechanisms of producer self-protection (immunity) have been predicted for the circular peptides. The first involves a designated immunity peptide, the mode of action of which has not yet been elucidated. By heterologous expression of various deletion variants of the cir operon in E. faecalis, cirE was pinpointed as the gene essential for circularin immunity (Kemperman et al., 2003a). In the enterocin AS-48 gene cluster this role falls to as-48D1 (Martinez-Bueno et al., 1998), whilst for butyrivibriocin, $b v i E$ has been proposed as the likely candidate (Kalmokoff et al., 2003). The second immunity mechanism involves an ABC-transport system, which although not essential for immunity, appears necessary for expression of complete resistance to the bacteriocin. An operon consisting of cirGHI in the circularin A locus (Kemperman et al., 2003a), and as-48EFGH in the enterocin AS-48 locus (Diaz et al., 2003), encodes the ABCtransporters suggested to confer this additional protection. Divergently transcribed from the pBt136-140 locus were the genes pBt133-130, encoding components of an $\mathrm{ABC}$ transport system with weak similarity to BacGHI from the bacteriocin 21 locus (Berry et al., 2002). The presence of an additional ABC-transporter was not detected in the uberolysin locus; however, as with the other circular bacteriocins, the locus contains a gene that may function as the putative immunity gene $(u b l E)$. In addition, previous studies have shown that cirBD and $a s-48 C_{1} D$ are capable of conferring low levels of immunity (Kemperman et al., 2003a, Martinez-Bueno et al., 1998). Therefore, a similar protective mechanism may be provided by $u b l B D$ in strain 42.

Upstream (by 52 bp) of the uberolysin locus (Fig. 2) is an ORF with homology to a purine biosynthesis gene (purHlike) from Streptococcus agalactiae (Tettelin et al., 2005). The final 159 bp of this ORF is duplicated, with the second copy (differing by only one bp) located $52 \mathrm{bp}$ downstream of $u b l E$. Thus, the $u b l$ locus appears to be flanked, $52 \mathrm{bp}$ from each end, by 159 bp direct repeats (Fig. 2). In the 52 bp 

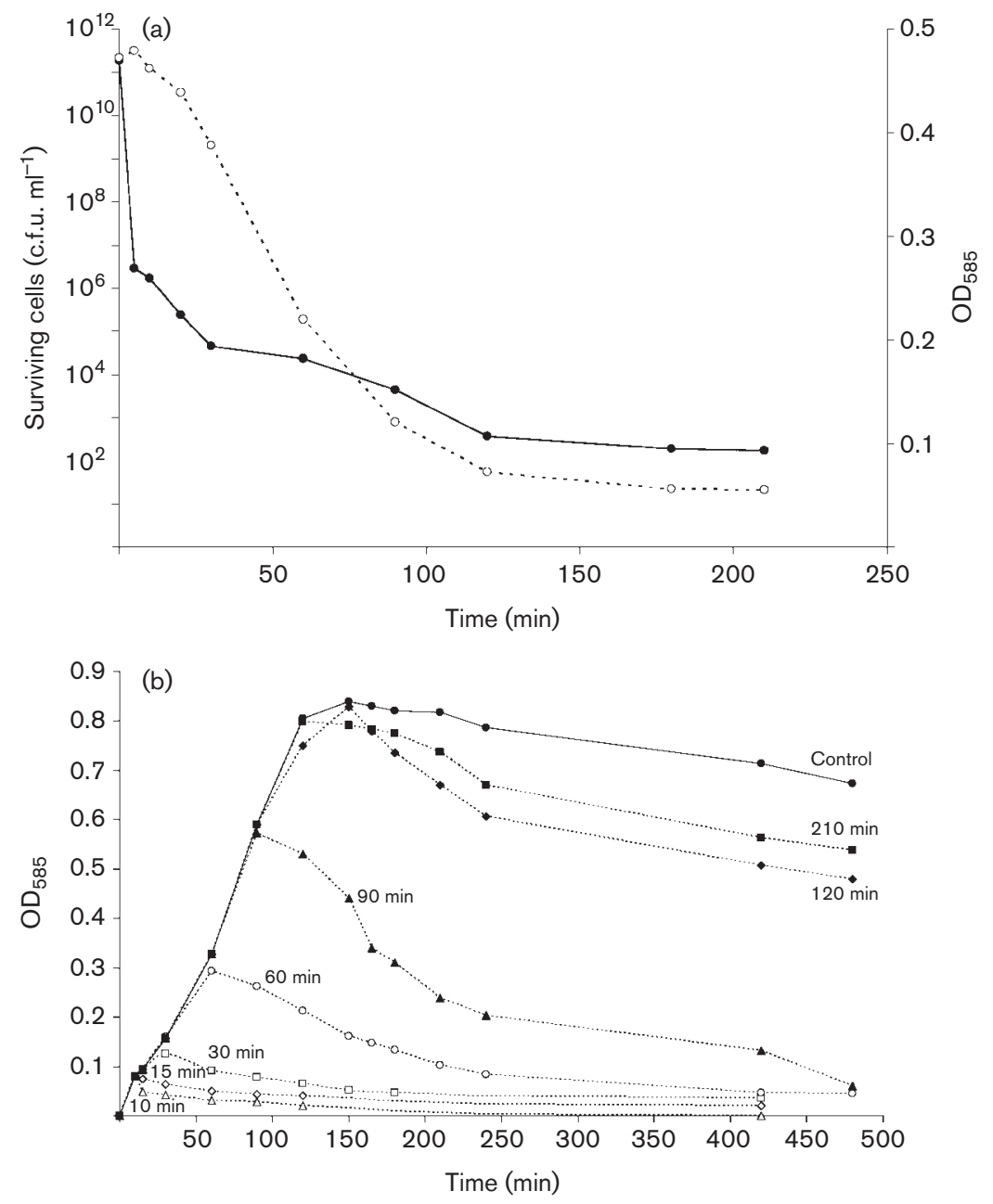

Fig. 3. The bactericidal and bacteriolytic nature of uberolysin. (a) Effect of uberolysin on the optical density $(\bigcirc)$ and viability $(\bullet)$ of a mid-exponential phase culture of $L$. lactis A5. Uberolysin (1 AU) was added at time point 0 . (b) Effect of uberolysin on growing cultures of $L$. lactis strain A5 when added at different phases of growth. Uberolysin $\left.(1 \mathrm{AU} \mathrm{ml})^{-1}\right)$ was added to a freshly inoculated culture after $10 \mathrm{~min}(\triangle)$, $15 \mathrm{~min}(\diamond), \quad 30 \mathrm{~min}(\square), \quad 60 \mathrm{~min}(\bigcirc)$, $90 \mathrm{~min}(\boldsymbol{\Delta}), 120 \mathrm{~min}(\boldsymbol{\Delta})$ or $210 \mathrm{~min}(\boldsymbol{\square})$ of incubation at $37^{\circ} \mathrm{C}$ and growth of the indicator strain was followed by measuring $\mathrm{OD}_{585} ; \boldsymbol{\bullet}$, control culture to which no uberolysin was added. intergenic region between the purH homologue and orf1 is a palindromic sequence, which could function as a bidirectional, rho-independent transcription terminator. An ORF with homologies to the GntR/MarR family of transcriptional regulators is located 542 bp downstream of the locus, and the intervening region contains short segments with similarity to S. agalactiae, Streptococcus pyogenes, and Streptococcus mutans genome sequences. The presence of these two, large (159 bp) direct repeats may indicate a possible method of acquisition of the locus by long target duplication. This mechanism for gene movement has been suggested for several microorganisms based on analysis and comparison of their complete genome sequences (Nobusato et al., 2000; Osaki et al., 2002; Xu et al., 2000). The 159 bp repeats found in $S$. uberis strain 42 were also found in $S$. uberis $0140 \mathrm{~J}$, with only three mismatches between both sets of repeats in these strains. This observation indicates that the locus may have been acquired by $S$. uberis early in its evolution as a species, rather than only by a particular strain of S. uberis. Interestingly, the loci for many of the circular bacteriocins are plasmid-borne (Martinez-Bueno et al., 1990; Tomita et al., 1997; Zheng et al., 2000), including that of the putative peptide antibiotic encoded by $p$ Bt136 (Berry et al., 2002). Since the latter is the only bacteriocin with which uberolysin shares weak similarity, it is tempting to speculate that the uberolysin locus may originally have been plasmid-associated prior to its incorporation into the $S$. uberis chromosome.

\section{The distribution of the uberolysin biosynthetic locus in S. uberis}

From a total of 45 additional S. uberis strains that were assessed, eight $(18 \%)$ were PCR positive for $u b l A$. Interestingly, one of those found to carry $u b l A$ was strain 0140J, the complete genome sequence of which is now publicly available (http://www.sanger.ac.uk/Projects/ S_uberis/). A BLAST search showed that strain 0140J also has the full complement of uberolysin locus genes, as well as the $159 \mathrm{bp}$ flanking direct repeats present in strain 42. However, two separate (PCR-confirmed) base pair changes introduce stop codons in orf1 of strain 0140J, probably resulting in formation of a truncated translational product. Moreover, no uberolysin-like activity could be detected from this strain. On the other hand, S. uberis strain 233, another producer of uberolysin, has an intact copy of orf 1 , indicating 
Table 3. Spectrum of inhibitory activity in deferred antagonism tests, and sensitivity to nisin $U$ and uberolysin, of $S$. uberis strains 42 and ublyko1

\begin{tabular}{|c|c|c|c|c|c|}
\hline \multirow[t]{2}{*}{ Bacterium } & \multirow[t]{2}{*}{$\begin{array}{c}\text { Strain } \\
\text { designation }\end{array}$} & \multicolumn{2}{|c|}{$\begin{array}{l}\text { Sensitivity in deferred antagonism tests } \\
\text { when tested against } S \text {. uberis strain: }\end{array}$} & \multicolumn{2}{|c|}{ Sensitivity to preparations of: } \\
\hline & & 42 & ublyko1* & Nisin $\mathrm{U} \dagger$ & Uberolysin \\
\hline \multirow[t]{3}{*}{ Enterococcus faecalis } & 96 & + & + & + & + \\
\hline & 97 & + & - & - & + \\
\hline & 98 & + & - & - & + \\
\hline \multirow[t]{2}{*}{ E. hirae } & ATCC 9790 & + & + & + & + \\
\hline & Lyt14\$ & + & - & - & + \\
\hline \multirow[t]{2}{*}{ Lactococcus lactis } & A5 & + & - & - & + \\
\hline & $\mathrm{T}-21$ & + & + & + & + \\
\hline L. innocua & L49 & + & - & - & + \\
\hline \multirow[t]{3}{*}{ Streptococcus agalactiae } & 76 & + & + & + & + \\
\hline & 77 & + & + & + & + \\
\hline & 78 & + & - & - & + \\
\hline S. anginosus & $\mathrm{T}-29$ & + & + & + & + \\
\hline \multirow[t]{3}{*}{ S. dysgalactiae } & 60 & + & + & + & + \\
\hline & 61 & + & + & + & + \\
\hline & 62 & + & + & + & + \\
\hline S. equisimilis & $\mathrm{T}-148$ & + & + & + & + \\
\hline \multirow[t]{2}{*}{ S. pyogenes } & FF22 & + & + & + & + \\
\hline & $71-679$ & + & + & + & + \\
\hline \multirow{2}{*}{ S. uberis } & $\mathrm{J} 4$ & + & - & - & + \\
\hline & J9 & + & + & + & + \\
\hline
\end{tabular}

*Uberolysin-negative mutant of strain 42 obtained by Tn916 mutagenesis.

$\dagger$ As described by Wirawan et al. (2006).

$\ddagger$ Vancomycin-resistant.

§Autolysin-deficient derivative of strain ATCC 9790 (Cornett et al., 1978).

\#Meticillin-resistant.

that orf1 may be essential for uberolysin production. Similarly, there have been independent reports of purification or partial purification from E. faecalis and E. faecium strains of molecules very similar to enterocin AS- 48 (Maisnier-Patin et al., 1996; Joosten et al., 1996; Tomita et al., 1997; Folli et al., 2003; Abriouel et al., 2005), indicating that this bacteriocin may be widespread amongst the enterococci. Indeed, a study based on PCR amplification with enterocin AS-48-specific primers detected the structural gene in 11 of 15 bacteriocin-producing enterococci (Joosten et al., 1997).

\section{Biological activity of uberolysin}

Addition of uberolysin to a mid-exponential-phase culture of $L$. lactis A5 caused immediate cessation of growth, followed by a 6-log decrease in viable cell numbers after only $10 \mathrm{~min}$ exposure (Fig. 3a), indicating it has a bactericidal 
mode of action. Concomitant assessment of the optical density of the culture demonstrated that uberolysin is also bacteriolytic. This ability of uberolysin to evoke bacteriolysis, either directly or indirectly, was the basis for it being named uberolysin. Moreover, addition of the bacteriocin to sensitive cultures at different phases of growth indicated that the target cells need to be actively dividing in order to undergo bacteriolysis, with early or mid-exponential growth phase cells being particularly susceptible (Fig. 3b). A similar effect has also been reported for enterocin AS-48, where immediate cessation of growth and a steady decrease in cell viability of E. faecalis S-47 occurred, followed by loss of turbidity (Maqueda et al., 2004). Whereas the turbidity decrease occurred 1-2 $\mathrm{h}$ after addition of enterocin AS-48 (Galvez et al., 1990), the corresponding effect of uberolysin on L. lactis A5 cells occurred within $30 \mathrm{~min}$. A similar effect results from the combined action of lactococcins A, B and $\mathrm{M}$, with death of $99.98 \%$ of sensitive cells occurring within the first $10 \mathrm{~min}$ of exposure, followed by a slower rate of bacteriolysis (Morgan et al., 1995). However, in contrast to enterocin AS-48, which causes lysis of E. faecalis cultures regardless of the growth phase (Galvez et al., 1990), uberolysin had only minimal effect on stationary-phase $L$. lactis cultures.

The bacteriolytic effect of uberolysin was also demonstrated against sensitive cells in agar medium (data not shown). Non-replicating cells appeared to be resistant to this lytic effect, as no zones of lysis were observed following the addition of uberolysin to heat- or chloroform-killed cells embedded in agar. Joosten et al. (1996) demonstrated that an enterocin AS-48 homologue, enterocin 4, had no effect on starved cells, but caused a marked decrease in the viability of energized cells. Moreover, some enterocin AS-48sensitive indicators did not lyse, even when considerably higher concentrations of peptide were used, indicating that bacteriolysis was likely to be a secondary effect on the cytoplasmic membrane, possibly as a result of topological activation of cell wall autolysins in the sensitive cells (Maqueda et al., 2004).

Uberolysin has a broad spectrum of inhibitory activity. Susceptible bacteria include most streptococci (with the exception of Streptococcus rattus and S. mutans), Listeria spp., enterococci and staphylococci (Table 3). However, Gram-negative bacteria do not appear to be susceptible to the action of uberolysin (data not shown). The spectra of inhibitory activity of S. uberis strain 42 and its uberolysinnegative derivative, strain ublyko1, were compared in deferred antagonism tests against a variety of indicator bacteria and these were compared with the antimicrobial activity of purified preparations of uberolysin and nisin $\mathrm{U}$ (Table 3). Taken together, the combined inhibitory activities of both uberolysin and nisin $U$ appears to account for the entire deferred antagonism spectrum of $S$. uberis strain 42 , indicating that, under the conditions employed, these two disparate bacteriocins account for the complete repertoire of bacteriocin production in strain 42 .

\section{Conclusion}

In the present study we demonstrate that $S$. uberis strain 42 , previously found to produce the lantibiotic nisin $U$, also produces a novel circular bacteriocin, uberolysin. Although uberolysin bears no primary sequence similarity to any of the currently known circular bacteriocins, it does share the defining characteristic of head-to-tail linkage of its $\mathrm{N}$ - and C-terminal amino acids after removal of a short leader peptide. Moreover, uberolysin is the first circular bacteriocin characterized from the genus Streptococcus.

\section{ACKNOWLEDGEMENTS}

This study was supported by research grants from the University of Otago (to J. R. T.), the University of Otago Research Grants Committee (to R. W. J.) and the Otago Medical Research Foundation (to R. W. J.), and with equipment grants provided by the New Zealand Lottery Grants Board (Lottery Health) and the Otago School of Medical Sciences (to R.W. J.). A University of Otago Publishing Award (to R.E.W.) was provided by the University of Otago Research Committee. In addition we thank the staff of the Protein Microchemistry Facility (Department of Biochemistry, University of Otago) for expert assistance with MS and automated N-terminal sequence analyses, and Dr N. C. K. Heng for critical discussions regarding the manuscript.

\section{REFERENCES}

Abriouel, H., Lucas, R., Ben Omar, N., Valdivia, E., Maqueda, M., Martinez-Canamero, M. \& Galvez, A. (2005). Enterocin AS48RJ: a variant of enterocin AS-48 chromosomally encoded by Enterococcus faecium RJ16 isolated from food. Syst Appl Microbiol 28, 383-397.

Altschul, S. F., Madden, T. L., Schaffer, A. A., Zhang, J., Zhang, Z., Miller, W. \& Lipman, D. J. (1997). Gapped BLAST and PSI-BLAST: a new generation of protein database search programs. Nucleic Acids Res 25, 3389-3402.

Berry, C., O'Neil, S., Ben-Dov, E., Jones, A. F., Murphy, L., Quail, M. A., Holden, M. T. G., Harris, D., Zaritsky, A. \& Parkhill, J. (2002). Complete sequence and organization of pBtoxis, the toxin-coding plasmid of Bacillus thuringiensis subsp. israelensis. Appl Environ Microbiol 68, 5082-5095.

Blowey, R. \& Edmondson, P. (1995). Mastitis Control in Dairy Herds: an Illustrated and Practical Guide. Ipswich, UK: Farming Press Books.

Buddle, B. M., Tagg, J. R. \& Ralston, M. J. (1988). Use of an inhibitor typing scheme to study the epidemiology of Streptococcus uberis mastitis. $N Z$ Vet J 36, 115-119.

Cornett, J. B., Redman, B. E. \& Shockman, G. D. (1978). Autolytic defective mutant of Streptococcus faecalis. J Bacteriol 133, 631-640.

Cotter, P. D., Hill, C. \& Ross, R. P. (2005). Bacteriocins: developing innate immunity for food. Nat Rev Microbiol 3, 777-788.

Craik, D. J., Daly, N. L., Saska, I., Trabi, M. \& Rosengren, K. J. (2003). Structures of naturally occurring circular proteins from bacteria. J Bacteriol 185, 4011-4021.

Cullen, G. A. (1966). The ecology of Streptococcus uberis. Br Vet J 122, 333-339.

Cullen, G. A. (1969). Streptococcus uberis: a review. Vet Bull 39, 155-165. 
de Greeff, A., Buys, H., van Alphen, L. \& Smith, H. E. (2002). Response regulator important in pathogenesis of Streptococcus suis serotype 2. Microb Pathog 33, 185-192.

Diaz, M., Valdivia, E., Martinez-Bueno, M., Fernandez, M., Soler-Gonzalez, A. S., Ramirez-Rodrigo, H. \& Maqueda, M. (2003). Characterization of a new operon, as-48EFGH, from the as-48 gene cluster involved in immunity to enterocin AS-48. Appl Environ Microbiol 69, 1229-1236.

Diep, D. B. \& Nes, I. F. (2002). Ribosomally synthesized antibacterial peptides in Gram positive bacteria. Curr Drug Targets 3, 107-122.

Folli, C., Ramazzina, I., Arcidiaco, P., Stoppini, M. \& Berni, R. (2003). Purification of bacteriocin AS-48 from an Enterococcus faecium strain and analysis of the gene cluster involved in its production. FEMS Microbiol Lett 221, 143-149.

Galvez, A., Maqueda, M., Valdivia, E., Quesada, A. \& Montoya, E. (1986). Characterization and partial purification of a broad spectrum antibiotic AS-48 produced by Streptococcus faecalis. Can J Microbiol 32, 765-771.

Galvez, A., Valdivia, E., Martinez-Bueno, M. \& Maqueda, M. (1990). Induction of autolysis in Enterococcus faecalis S-47 by peptide AS-48. J Appl Bacteriol 69, 406-413.

Hancock, L. \& Perego, M. (2002). Two-component signal transduction in Enterococcus faecalis. J Bacteriol 184, 5819-5825.

Haydel, S. E. \& Clark-Curtiss, J. E. (2004). Global expression analysis of two-component system regulator genes during Mycobacterium tuberculosis growth in human macrophages. FEMS Microbiol Lett 236, 341-347.

Heng, N. C. K. \& Tagg, J. R. (2006). What's in a name? Class distinction for bacteriocins. Nat Rev Microbiol 4, available online at: http://www.nature.com/nrmicro/journal/v4/n2/full/nrmicro1273-cl.html.

Hickey, R. M., Twomey, D. P., Ross, R. P. \& Hill, C. (2003). Potential of the enterocin regulatory system to control expression of heterologous genes in Enterococcus. J Appl Microbiol 95, 390-397.

Hutchings, M. I., Hoskisson, P. A., Chandra, G. \& Buttner, M. J. (2004). Sensing and responding to diverse extracellular signals? Analysis of the sensor kinases and response regulators of Streptomyces coelicolor A3(2). Microbiology 150, 2795-2806.

Jack, R., Bierbaum, G. \& Sahl, H.-G. (1998). Lantibiotics and Related Peptides. Heidelberg: Springer.

Jayarao, B. M., Oliver, S. P., Tagg, J. R. \& Matthews, K. R. (1991). Genotypic and phenotypic analysis of Streptococcus uberis isolated from bovine mammary secretions. Epidemiol Infect 107, 543-555.

Joosten, H. M., Nunez, M., Devreese, B., Van Beeumen, J. \& Marugg, J. D. (1996). Purification and characterization of enterocin 4, a bacteriocin produced by Enterococcus faecalis INIA 4. Appl Environ Microbiol 62, 4220-4223.

Joosten, H. M., Rodriguez, E. \& Nunez, M. (1997). PCR detection of sequences similar to the AS-48 structural gene in bacteriocinproducing enterococci. Lett Appl Microbiol 24, 40-42.

Kabuki, T., Saito, T., Kawai, Y., Uemura, J. \& Itoh, T. (1997). Production, purification and characterization of reutericin 6, a bacteriocin with lytic activity produced by Lactobacillus reuteri LA6. Int J Food Microbiol 34, 145-156.

Kalmokoff, M. L. \& Teather, R. M. (1997). Isolation and characterization of a bacteriocin (Butyrivibriocin AR10) from the ruminal anaerobe Butyrivibrio fibrisolvens AR10: evidence in support of the widespread occurrence of bacteriocin-like activity among ruminal isolates of $B$. fibrisolvens. Appl Environ Microbiol 63, 394-402.

Kalmokoff, M. L., Cyr, T. D., Hefford, M. A., Whitford, M. F. \& Teather, R. M. (2003). Butyrivibriocin AR10, a new cyclic bacteriocin produced by the ruminal anaerobe Butyrivibrio fibrisolvens AR10: characterization of the gene and peptide. Can J Microbiol 49, 763-773.
Kawai, Y., Saito, T., Kitazawa, H. \& Itoh, T. (1998a). Gassericin A; an uncommon cyclic bacteriocin produced by Lactobacillus gasseri LA39 linked at N- and C-terminal ends. Biosci Biotechnol Biochem 62, 2438-2440.

Kawai, Y., Saito, T., Suzuki, M. \& Itoh, T. (1998b). Sequence analysis by cloning of the structural gene of gassericin A, a hydrophobic bacteriocin produced by Lactobacillus gasseri LA39. Biosci Biotechnol Biochem 62, 887-892.

Kawai, Y., Ishii, Y., Uemura, K., Kitazawa, H., Saito, T. \& Itoh, T. (2001). Lactobacillus reuteri LA6 and Lactobacillus gasseri LA39 isolated from the faeces of the same human infant produce identical cyclic bacteriocin. Food Microbiol 18, 407-415.

Kawai, Y., Arakawa, K., Itoh, A., Saitoh, B., Ishii, Y., Nishimura, J., Kitazawa, H., Itoh, T. \& Saito, T. (2003). Heterologous expression of gassericin A, a bacteriocin produced by Lactobacillus gasseri LA39. Anim Sci J 74, 45-51.

Kawai, Y., Ishii, Y., Arakawa, K., Uemura, K., Saitoh, B., Nishimura, J., Kitazawa, H., Yamazaki, Y., Tateno, Y. \& other authors (2004a). Structural and functional differences in two cyclic bacteriocins with the same sequences produced by lactobacilli. Appl Environ Microbiol 70, 2906-2911.

Kawai, Y., Kemperman, R., Kok, J. \& Saito, T. (2004b). The circular bacteriocins gassericin A and circularin A. Curr Protein Pept Sci 5, 393-398.

Kawulka, K. E., Sprules, T., Diaper, C. M., Whittal, R. M., McKay, R. T., Mercier, P., Zuber, P. \& Vederas, J. C. (2004). Structure of subtilosin A, a cyclic antimicrobial peptide from Bacillus subtilis with unusual sulfur to alpha-carbon cross-links: formation and reduction of alpha-thio-alpha-amino acid derivatives. Biochemistry 43, 3385-3395.

Kemperman, R., Jonker, M., Nauta, A., Kuipers, O. P. \& Kok, J. (2003a). Functional analysis of the gene cluster involved in production of the bacteriocin circularin A by Clostridium beijerinckii ATCC 25752. Appl Environ Microbiol 69, 5839-5848.

Kemperman, R., Kuipers, A., Karsens, H., Nauta, A., Kuipers, O. \& Kok, J. (2003b). Identification and characterization of two novel clostridial bacteriocins, circularin A and closticin 574. Appl Environ Microbiol 69, 1589-1597.

Khan, I. U., Hassan, A. A., Abdulmawjood, A., Lammler, C., Wolter, W. \& Zschock, M. (2003). Identification and epidemiological characterization of Streptococcus uberis isolated from bovine mastitis using conventional and molecular methods. J Vet Sci 4, 213-224.

Klaenhammer, T. R. (1993). Genetics of bacteriocins produced by lactic acid bacteria. FEMS Microbiol Rev 12, 39-85.

Klesse, N. A. (2001). Purification and partial characterisation of a novel bacteriocin produced by Streptococcus uberis strain ATCC 27958. MSc thesis, University of Otago, Dunedin.

Knutsen, E., Ween, O. \& Håvarstein, L. S. (2004). Two separate quorum-sensing systems upregulate transcription of the same $\mathrm{ABC}$ transporter in Streptococcus pneumoniae. J Bacteriol 186, 3078-3085.

Leigh, J. A. (1999). Streptococcus uberis: a permanent barrier to the control of bovine mastitis? Vet J 157, 225-238.

Maisnier-Patin, S., Forni, E. \& Richard, J. (1996). Purification, partial characterisation and mode of action of enterococcin EFS2, an antilisterial bacteriocin produced by a strain of Enterococcus faecalis isolated from a cheese. Int J Food Microbiol 30, 255-270.

Maqueda, M., Galvez, A., Bueno, M. M., Sanchez-Barrena, M. J., Gonzalez, C., Albert, A., Rico, M. \& Valdivia, E. (2004). Peptide AS48: prototype of a new class of cyclic bacteriocins. Curr Protein Pept Sci 5, 399-416.

Martinez-Bueno, M., Galvez, A., Valdivia, E. \& Maqueda, M. (1990). A transferable plasmid associated with AS-48 production in Enterococcus faecalis. J Bacteriol 172, 2817-2818. 
Martinez-Bueno, M., Maqueda, M., Galvez, A., Samyn, B., Van Beeumen, J., Coyette, J. \& Valdivia, E. (1994). Determination of the gene sequence and the molecular structure of the enterococcal peptide antibiotic AS-48. J Bacteriol 176, 6334-6339.

Martinez-Bueno, M., Valdivia, E., Galvez, A., Coyette, J. \& Maqueda, M. (1998). Analysis of the gene cluster involved in production and immunity of the peptide antibiotic AS-48 in Enterococcus faecalis. Mol Microbiol 27, 347-358.

Morgan, S., Ross, R. P. \& Hill, C. (1995). Bacteriolytic activity caused by the presence of a novel lactococcal plasmid encoding lactococcins A, B, and M. Appl Environ Microbiol 61, 2995-3001.

Nes, I. F. \& Tagg, J. R. (1996). Novel lantibiotics and their prepeptides. Antonie van Leeuwenhoek 69, 89-97.

Nida, K. \& Cleary, P. P. (1983). Insertional inactivation of streptolysin S expression in Streptococcus pyogenes. J Bacteriol 155, 1156-1161.

Nobusato, A., Uchiyama, I., Ohashi, S. \& Kobayashi, I. (2000). Insertion with long target duplication: a mechanism for gene mobility suggested from comparison of two related bacterial genomes. Gene 259, 99-108.

Osaki, M., Takamatsu, D., Shimoji, Y. \& Sekizaki, T. (2002). Characterization of Streptococcus suis genes encoding proteins homologous to sortase of gram-positive bacteria. J Bacteriol 184, 971-982.

Sahl, H.-G. \& Bierbaum, G. (1998). Lantibiotics: biosynthesis and biological activities of uniquely modified peptides from grampositive bacteria. Annu Rev Microbiol 52, 41-79.

Sambrook, J. \& Russell, D. W. (2001). Molecular Cloning: a Laboratory Manual, 3rd edn. Cold Spring Harbor, NY: Cold Spring Harbor Laboratory.

Shevchenko, A., Jensen, O. N., Podtelejnikov, A. V., Sagliocco, F., Wilm, M., Vorm, O., Mortensen, P., Boucherie, H. \& Mann, M. (1996). Linking genome and proteome by mass spectrometry: large-scale identification of yeast proteins from two dimensional gels. Proc Natl Acad Sci U S A 93, 14440-14445.
Tagg, J. R. \& Bannister, L. V. (1979). "Fingerprinting" betahaemolytic streptococci by their production of and sensitivity to bacteriocine-like inhibitors. J Med Microbiol 12, 397-411.

Tagg, J. R. \& Vugler, L. G. (1986). An inhibitor typing scheme for Streptococcus uberis. J Dairy Res 53, 451-456.

Tagg, J. R., Dajani, A. S. \& Wannamaker, L. W. (1976). Bacteriocins of gram-positive bacteria. Bacteriol Rev 40, 722-756.

Tettelin, H., Masignani, V., Cieslewicz, M. J., Donati, C., Medini, D., Ward, N. L., Angiuoli, S. V., Crabtree, J., Jones, A. L. \& other authors (2005). Genome analysis of multiple pathogenic isolates of Streptococcus agalactiae: implications for the microbial "pangenome". Proc Natl Acad Sci U S A 102, 13950-13955.

Tomita, H., Fujimoto, S., Tanimoto, K. \& Ike, Y. (1997). Cloning and genetic and sequence analyses of the bacteriocin 21 determinant encoded on the Enterococcus faecalis pheromone-responsive conjugative plasmid pPD1. J Bacteriol 179, 7843-7855.

Ulijasz, A. T., Andes, D. R., Glasner, J. D. \& Weisblum, B. (2004). Regulation of iron transport in Streptococcus pneumoniae by RitR, an orphan response regulator. J Bacteriol 186, 8123-8136.

Wescombe, P. A. \& Tagg, J. R. (2003). Purification and characterization of streptin, a type Al lantibiotic produced by Streptococcus pyogenes. Appl Environ Microbiol 69, 2737-2747.

Wirawan, R. E., Klesse, N. A., Jack, R. W. \& Tagg, J. R. (2006). Molecular and genetic characterization of a novel nisin variant produced by Streptococcus uberis. Appl Environ Microbiol 72, 1148-1156.

Xu, Q., Stickel, S., Roberts, R. J., Blaser, M. J. \& Morgan, R. D. (2000). Purification of the novel endonuclease, Hpy188I, and cloning of its restriction-modification genes reveal evidence of its horizontal transfer to the Helicobacter pylori genome. J Biol Chem 275, 17086-17093.

Zheng, G., Hehn, R. \& Zuber, P. (2000). Mutational analysis of the sbo-alb locus of Bacillus subtilis: identification of genes required for subtilosin production and immunity. J Bacteriol 182, 3266-3273.

Edited by: D. M. Gordon 